\title{
Caravan-Submm, Black Hole Imager in the Andes
}

\author{
Makoto Miyoshi, ${ }^{1}$ Takashi Kasuga, ${ }^{2}$ Jose K. Ishitsuka Iba, ${ }^{3}$ \\ Tomoharu Oka, ${ }^{4}$ Mamoru Sekido, ${ }^{5}$ Kazuhiro Takefuji, ${ }^{5}$ Masaaki Takahashi, ${ }^{6}$ \\ Hiromi Saida, ${ }^{7}$ and Rohta Takahashi ${ }^{8}$ \\ ${ }^{1}$ National Astronomical Observatory, 2-21-1 Osawa, Mitaka, Tokyo 181-8588, Japan \\ ${ }^{2}$ Department of Advanced Sciences, Faculty of Science and Engineering, Hosei University, 3-7-2 Kajino, \\ Koganei, Tokyo 184-8584, Japan \\ ${ }^{3}$ Geophysical Institute of Peru, Carretera Ing. Alberto A. Giesecke M. Km 15, Huachac, Peru \\ ${ }^{4}$ Department of Physics, Institute of Science and Technology, Keio University, 3-14-1 Hiyoshi, Yokohama, Kanagawa 223-8522, Japan \\ ${ }^{5}$ Kashima Space Technology Center, National Institute of Information and Communications Technology, 893-1 Hirai, \\ Kashima, Ibaraki 314-8501, Japan \\ ${ }^{6}$ Department of Physics and Astronomy, Aichi University of Education, Kariya 448-8542, Japan \\ ${ }^{7}$ Department of Physics, Daido University, Minami-ku, Nagoya 457-8530, Japan \\ ${ }^{8}$ National Institute of Technology, Tomakomai College, 443 Nishikioka, Tomakomai, Hokkaido 059-1275, Japan \\ Correspondence should be addressed to Makoto Miyoshi; makoto.miyoshi@nao.ac.jp
}

Received 3 March 2016; Accepted 16 June 2016

Academic Editor: Roberto Turolla

Copyright (C) 2016 Makoto Miyoshi et al. This is an open access article distributed under the Creative Commons Attribution License, which permits unrestricted use, distribution, and reproduction in any medium, provided the original work is properly cited.

\begin{abstract}
Imaging a black hole horizon as a shadow at the center of black hole accretion disk is another method to prove/check Einstein's general relativity at strong gravitational fields. Such black hole imaging is expected to be achievable using a submillimeter wavelength VLBI (very long baseline interferometer) technique. Here, we introduce a Japanese black hole imaging project, Caravansubmm undertaken in the Andes.
\end{abstract}

\section{Introduction}

1.1. Black Hole Shadow, Evidence of a Black Hole. The detection of gravitational waves from merging a pair of black holes is the final verification of Einstein's general relativity [1]. The detection of these also opens a new field so called black hole astronomy. Also, imaging a black hole horizon as a shadow at the center of a black hole accretion disk is an independent method to prove/check Einstein's general relativity at strong gravitational fields. Because the vicinity of the horizon is a "strong" gravitational field, observations of behaviors of electromagnetic waves around a black hole must be the best laboratory for general relativity and theories of gravity. The black hole itself is entirely pitch black, but around a black hole, matter that falls onto the black hole forms a very hot disk, where strong electromagnetic waves are emitted. Black hole horizons can be observed as shadows at the centers of such bright and hot accretion disks (Figure 1).

Views of black holes in such situations have been theoretically investigated by many theorists [2-6]. From the images of the black hole shadows, the physical parameters of black holes, that is, mass, spin, and charge, can be determined without degeneracies between these parameters (Figure 2). In other words, precise measurement of the shadow shape gives us complete information of the black hole.

In this paper we introduce that imaging a black hole as a shadow is on the verge of being accomplished after several issues are resolved. First we explain the best target source is Sagittarius $A^{*}\left(\operatorname{SgrA}^{*}\right)$, the Galactic Center massive black hole in Section 1.2. Second we explain several points that 


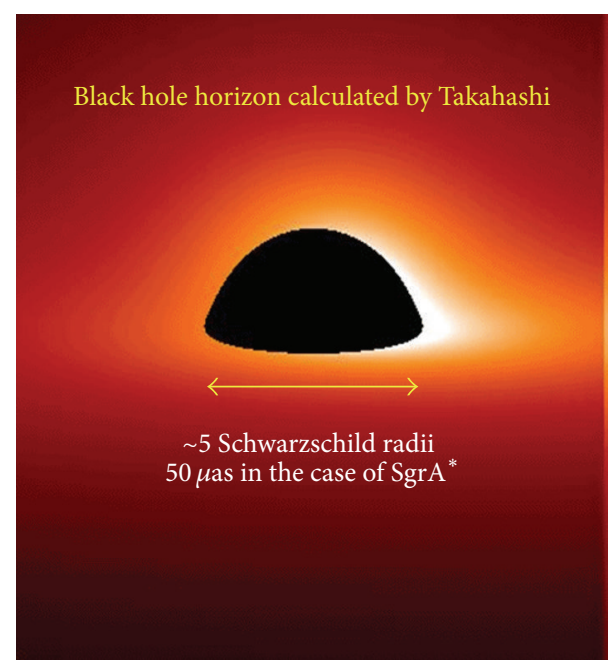

Figure 1: Image of black hole shadow calculated by Takahashi [7]. The black hole shadow size in $\operatorname{SgrA}^{*}$ is about $50 \mu$ as in diameter.
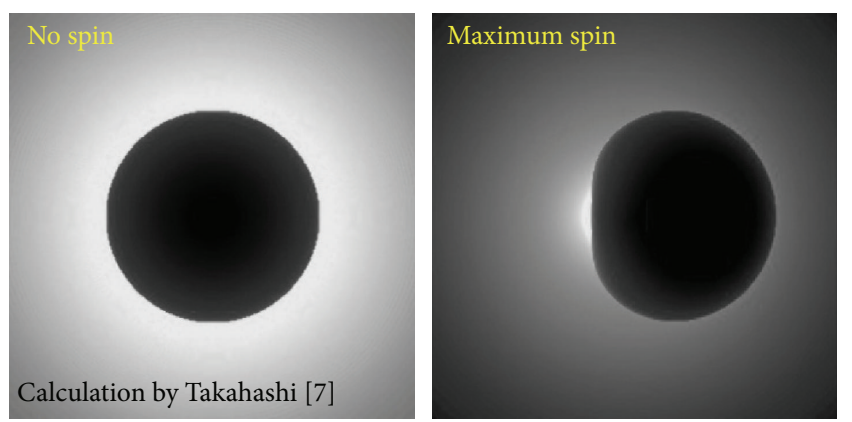

FIGURE 2: Effect of black hole rotation (spin effect). The shape of black hole horizon is distorted by the frame dragging. By measuring the aspect ratio we can investigate the spin of black hole Takahashi [7].

should be settled in Section 1.3. Then in Sections 2 and 3 we explain our proposed plan, Caravan-submm, a black hole imager in the Andes will help settle these issues.

1.2. The Leading Target Source, Sagittarius $A^{*}$. Considering the feasibility of imaging the black hole horizon, the apparent angular size of the Schwarzschild radius is the first critical factor to consider. It is almost impossible to gain an understanding of the sizes and figures of stellar black holes through observations. In the case of a stellar black hole located at $1 \mathrm{pc}\left(1 \mathrm{pc}=3.086 \times 10^{13} \mathrm{~km}\right.$, or 3.26 lightyears $)$ with $1 \mathrm{M}_{\odot}$, the Schwarzschild radius, $2.95 \times 10^{3} \mathrm{~m}$, corresponds to only $0.02 \mu$ arc seconds ( $\mu$ as) in angular size (shadow size is $0.1 \mu \mathrm{as}$ ). This angular size is too small to distinguish with spatial resolution of present or near future telescopes.

Because the Schwarzschild radius is proportional to the mass of black hole and also because the apparent angular size is inversely proportional to the distance, super massive black holes at relatively short distance are much more promising to observe. Among them, the most promising target source is Sagittarius $A^{*}\left(\operatorname{SgrA}^{*}\right)$, the Galactic Center massive black hole $(\mathrm{GCBH})$ with mass $M_{\mathrm{GC}} \sim 4 \times 10^{6} \mathrm{M}_{\odot}$ [11]. Because of both the short distance from the earth $8 \mathrm{kpc}$ and the huge mass, the apparent angular size of its Schwarzschild radius is $10 \mu$ as. Further the mass of SgrA $^{*}$ is most precisely measured from motions of stars closely orbiting around SgrA*. Using the lower limit on the mass and the measured size $\sim 1 \mathrm{AU}$ (astronomical unit, $1.5 \times 10^{8} \mathrm{~km}$ ) at $86 \mathrm{GHz}$, the inferred density exceeds a $6.5 \times 10^{21} \mathrm{M}_{\odot} \mathrm{pc}^{-3}$; therefore, SgrA ${ }^{*}$ cannot be a kind of star cluster [12]. Today SgrA* is the most convincing object for a black hole. The black hole horizon or black hole shadow of $~ 50 \mu$ as in diameter can be observed using the VLBI technique [8, 13-15] (the next promising source is the super massive black hole at the center of M 87; the black hole mass is estimated to be $(6.6 \pm 0.4) \times 10^{9} \mathrm{M}_{\odot}$ by Gebhardt et al. [16]. Assuming the distance to be $16 \mathrm{Mpc}$, the apparent angular size of its Schwarzschild radius is $8 \mu$ as. The prominent jet of M87 may hinder the viewing of the black hole shadow).

1.3. Present Status of Viewing the Black Hole Shadow. With the expectation of observing the surroundings of a black hole, the structure of $\mathrm{SgrA}^{*}$ has been investigated using VLBI. However, it is now well known that the observed image of SgrA* is totally broadened and obscured due to the scattering effect of circumnuclear plasma at lower-frequency observations [12, 17-19]. This scattering effect occurs not along the long path to the sun on the galactic plane but mainly in the region within $150 \mathrm{pc}$ from the Galactic Center [20]. The same kinds of plasma regions probably exist in all Galactic Centers. Therefore, the situation will be the same for observing black holes at any active galactic nuclei.

Namely, how to cope with the scattering effect of surrounding plasma is the second factor to be considered. From multiple-frequency VLBI observations of SgrA* mainly using the VLBA it has emerged that the scattering effect shows a $\lambda^{2}$ dependence where $\lambda$ is an observing frequency. The intrinsic structure of SgrA* cannot be observed at lower frequencies, but at the higher observing frequencies, the scattering effect becomes smaller. It is expected that the intrinsic figure of SgrA $^{*}$ will appear at submillimeter wavelength VLBI observations [13].

The first VLBI observation of SgrA* above $200 \mathrm{GHz}$ was performed by Krichbaum et al. [9]; they estimated the size of SgrA* to be $110 \mu$ as from $1100 \mathrm{~km}$ baseline observations. After a decade, American research group (MIT, Haystack Observatory) succeeded in obtaining the first fringe detection using multistations from SgrA* observations at $230 \mathrm{GHz}$ [15]. They used a three-station VLBI array consisting of the $10 \mathrm{~m}$ Submillimeter Telescope Observatory (SMT) on Mt. Graham in Arizona, one 10 m element of the Combined Array for Research in Millimeter-Wave Astronomy (CARMA) in Eastern California, and the $15 \mathrm{~m}$ JCMT in Hawaii. They found fringes from projected baselines of $\sim 3.5 \times 10^{9} \lambda(4550 \mathrm{~km})$ with a fringe spacing of $60 \mu$ as. Though the data were not sufficient to make a synthesis image but were well fitted by a Gaussian brightness distribution with a full width half max 
size of $46.0 \mu$ as. Taking the still remaining scattering effect into account, the intrinsic size of $\operatorname{SgrA}^{*}$ at $230 \mathrm{GHz}$ was estimated to be $40 \mu$ as. They further achieved investigation of the magnetic field of SgrA* from their continued EHT (Event Horizon Telescope) project [21] but have still not obtained a sufficient data set to make a synthesis image of the SgrA* black hole.

There are two major reasons why imaging the SgrA ${ }^{*}$ black hole is still difficult. One is the insufficient $u-v$ coverage (amount of sampled data) to make a high quality synthesis image. Radio interferometers including VLBIs sample spatial Fourier components of the brightness distribution of observing source (in radio astronomy, we call the sampled spatial Fourier component visibility). Synthesis image of the source is obtained by inverse Fourier transform of the sampled data. Naturally, if the amount of sampling data is insufficient, it is difficult to obtain a high quality synthesis image. From the simulations Miyoshi et al. [8] found that suitable arrays for imaging the SgrA* black hole shadow at $230 \mathrm{GHz}$ require more than 10 stations located mainly in the southern hemisphere extending to the $8000 \mathrm{~km}$ region. The addition of stations in the northern hemisphere improves the image further. The present number of available radio telescopes is insufficient for SgrA* VLBI imaging at $230 \mathrm{GHz}$ or above.

The other main reason lies in data calibrations. Even at relatively lower frequencies of 43 or $86 \mathrm{GHz}$, calibrating VLBI data of SgrA* was known to be very difficult. From the experiences using the VLBA, Bower et al. [22] noted the following: (1) VLBI arrays were located in the northern hemisphere and lacked north-south spatial resolutions for $\operatorname{SgrA}^{*}\left(\delta=-30^{\circ}\right)$ to measure even the minor axis size of elliptical Gaussian shape assumed for SgrA*. (2) Observations of SgrA ${ }^{*}$ with such existing VLBI arrays had to be performed at low elevations and it is difficult to calibrate visibility sufficiently. Therefore, even at lower frequencies, it is why we had to abandon synthesis imaging of $\operatorname{SgrA}^{*}$ and to use a kind of model fitting to the data. Shen et al. [12] and Bower et al. [17] used the closure phase that is automatically free from systematic errors (sum of three visibility phases found from three baselines forming a triangle). Assuming elliptical Gaussian shapes as SgrA* structure; they fitted to closure quantities and estimated the size and the position angle of elongation of $\mathrm{SgrA}^{*}$. The main origin of such data errors is the atmospheric time variations that bring phase and amplitude variation of obtained spatial Fourier components of the SgrA $^{*}$ structure. The atmospheric time variations bring larger and more rapid fluctuations into data at higher observing frequencies. For imaging black holes we must cope with such errors and establish complete data calibration method.

1.4. Points at Issue. Here we summarize the issues and countermeasures in order to achieve imaging of black hole horizon. The first point is the apparent angular size of the Schwarzschild radius of the black hole. Because SgrA* has the largest one, it is the leading candidate for viewing of a black hole horizon. The second point is the observing frequency. The higher frequencies around $230 \mathrm{GHz}$ or above are required. This is not because of obtaining higher spatial resolutions but because of avoiding the scattering effect of plasma around (massive) black holes. The third is imaging capability of VLBI network we use. To obtain sufficient data for high quality synthesis imaging of the SgrA* black hole horizon, we need much VLBI stations as well as 10 stations mainly in the southern hemisphere. At present, particularly shorter baselines around from 1000 to $2000 \mathrm{~km}$ in length are missing.

As long as we perform observations on the ground, obtained data suffer the effects caused by the atmosphere. As for present data calibrations at millimeter/submillimeter VLBI on SgrA*, we have to give up making an effort for precise calibrations and are forced to use model fitting with closure quantities. However, without synthesis imaging using correctly calibrated data, it is almost impossible to find real structures of the source, and in particular, unexpected structures are never detected. Establishing a correct calibration method is a key for obtaining real images of black hole horizons.

As previously mentioned, we need new VLBI stations to sample sufficient data set. This means we need a budget for station construction. In order to achieve the construction of new VLBI stations immediately, cost reduction for construction is necessary. Because $\operatorname{SgrA}^{*}$ is an object located in the southern sky, to observe SgrA ${ }^{*}$ with fine observing condition, the southern hemisphere is desirable place for new VLBI stations. In addition, a better site for submillimeter wavelength observations is in the higher mountains where the amount of atmospheric water vapor is lower. It is in such a place that we can receive radio waves at submillimeter wavelength from the universe with low noise. The Andes is a suitable location. In the Andes there are other submillimeter telescopes including the ALMA, meaning it is also suitable for obtaining shorter baselines with new VLBI stations. In the next section, we introduce our proposed plan Caravansubmm in the Andes.

\section{Caravan-Submm in the Andes}

Japan has a dedicated plan for black hole imaging, named "Caravan-submm," which is a project to construct a millimeter/submillimeter wavelength VLBI network in the Andes. In 2012, we put forward the plan, Caravan-submm to the Astronomy and Astrophysics subcommittee of Science Council of Japan (SCJ), where proposed future plans (middle size projects) were discussed openly (http://www.scj.go.jp/ja/ member/iinkai/kiroku/3-140912.pdf. This report is written in Japanese only).

The new VLBI network contains at least its own two fixed VLBI stations and one mobile VLBI station. We plan to construct the fixed stations at around Huancayo Observatory, Geophysical Institute of Peru (IGP), and at the Mount Chacaltaya Laboratory in Bolivia. Huancayo Observatory is at Huancayo Province in Peru $\left(12.0^{\circ} \mathrm{S}, 75.29^{\circ} \mathrm{W}, 3370 \mathrm{~m}\right.$ in altitude), on the equator of the Earth's magnetic field. The observatory is famous for observation of geomagnetism. In collaboration with the National Astronomical Observatory of Japan (NAOJ), the director, Professor Ishitsuka, promotes radio astronomy with the Sicaya $32 \mathrm{~m}$ radio telescope. 

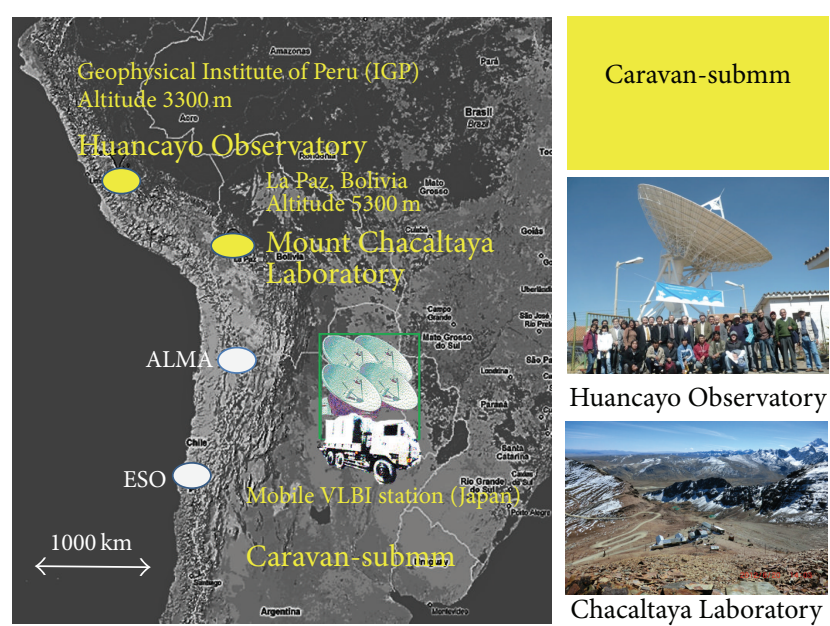

FIGURE 3: Caravan-submm in the Andes. The mobile radio telescope Caravan-submm moves around Andes.

The Mount Chacaltaya Laboratory is on the top of Mt. Chacaltaya, near La Paz in Bolivia, and is operated in collaboration with worldwide institutes $\left(16.35^{\circ} \mathrm{S}, 68.13^{\circ} \mathrm{W}, 5270 \mathrm{~m}\right.$ in altitude). It is at the Chacaltaya Laboratory that the Yukawa's predicted $\pi$-meson was detected in 1947 . The observatory's collaboration with Japan, namely, with the Institute for Cosmic Ray Research (ICRR) at the University of Tokyo, has been ongoing since 1962 (Figure 3).

2.1. Caravan-Submm, Mobile VLBI Station. For imaging black holes, as mentioned in the previous section, one of the key points is the $u-v$ coverage and how many spatial Fourier components of the observing source can be sampled. At present, it is essentially important to obtain shorter baselines around from 1000 to $2000 \mathrm{~km}$ in length for the black hole imaging of SgrA*. In order to sample sufficient VLBI data for good imaging, till now, we have made efforts to construct as many new VLBI stations as possible. However, instead, Caravan-submm includes one mobile VLBI station. By changing observational positions among Andes mountains, we repeat VLBI observations of SgrA* and sample various kinds of VLBI data which allow us to make high quality images with excellent cost performance. The shape of the black hole horizon shows a constant figure because the shape is defined only by the black hole space-time. Therefore it is no matter to take the picture with longer exposure time. We intend to make the mobile station first, prior to the constructions of fixed stations. The required baseline lengths ranging from 1000 to $2000 \mathrm{~km}$ can be realized not only by our own fixed stations but also by collaborating with nearby ALMA (an open use radio telescope) and/or other telescopes in the Andes.

A VLBI mobile station or transportable station is a famous method in Japan because such geodetic VLBI experiments have been performed since the mid-1980s [23]. Also the Geospatial Information Authority of Japan (GSI) performed geodetic VLBI project using VLBI mobile stations

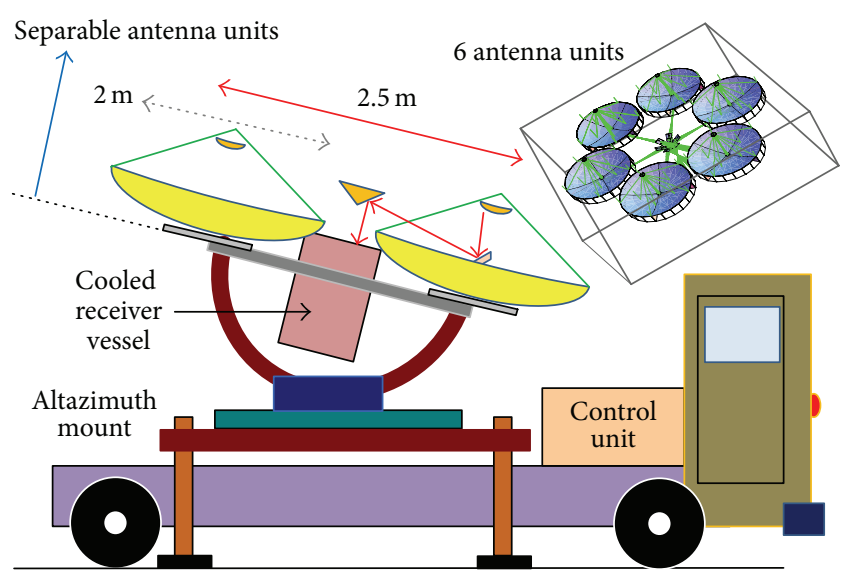

FIGURE 4: Caravan-submm, mobile VLBI station.

[24, 25]. Using the same method, we sample VLBI data effectively and intend to attain higher quality images at submillimeter VLBI observations than those only fixed stations can do.

For transportation, the Caravan-submm mobile station will contain at least three trucks: one for antennas, another for mount, and the other for the electric power supply, VLBI recording system, and hydrogen maser. However, we intend to obtain the power supply for telescope operations from commercial base (Figure 4). The Peruvian and Bolivian Andes are developed regions with many cities and towns, meaning that we can easily obtain a commercially based electric power supply. Main roads also have been well developed throughout the Andes, which means the Caravan-submm mobile station can quite easily be transported.

The difference is the observing frequency between the mobile station for geodetic VLBI yesteryear and our new Caravan mobile station. We have to receive $230 \mathrm{GHz}$ band while the old geodetic VLBI utilized SX bands $(2 \mathrm{GHz}$ and $8 \mathrm{GHz}$ ) for positional measurements. The higher the observing frequency we use, the more difficult the maintaining of coherency of observing system becomes. However, recent VLBI experiment at $230 \mathrm{GHz}$ in Japan provided us with technical evidence for the Caravan mobile station. At Nobeyama radio observatory in April 2015, using two radio telescopes with independent frequency standards, VLBI fringe at $230 \mathrm{GHz}$ was detected from a tentative set-up of VLBI system. It was the first VLBI fringe detection at $230 \mathrm{GHz}$ in Japan, which is the world tie record for the highest frequency VLBI [26]. This proves that the idea of Caravansubmm, mobile submillimeter telescope is technically feasible. The used VLBI system for that experiment was a very temporary one, composed of instruments the participants brought together in haste. This situation was very similar to that of the Caravan-submm mobile observing system.

2.2. Multimirror System for the Mobile Station. We must further elaborate ideas to realize a mobile $230 \mathrm{GHz}$ radio telescope, Caravan-submm mobile station. Today, following 


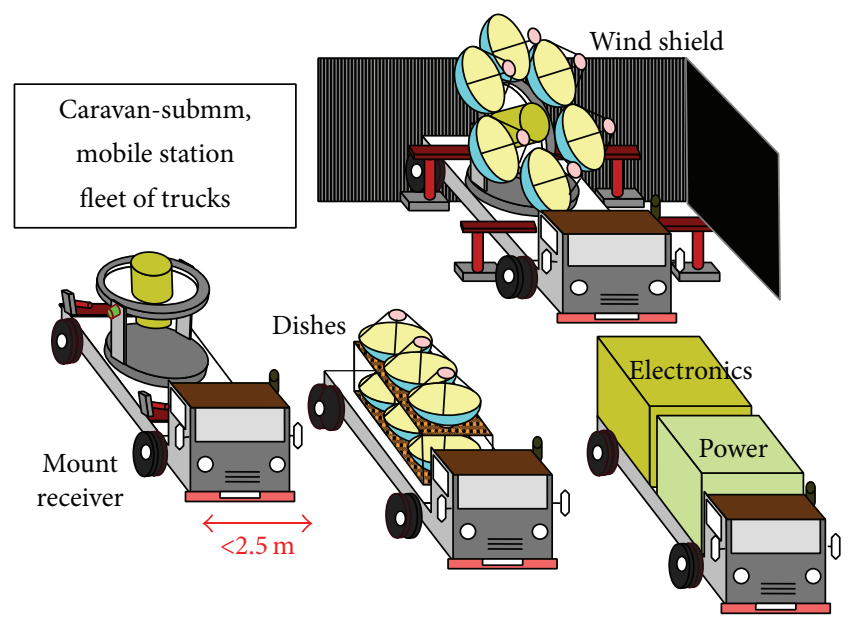

FIgURE 5: Caravan-submm, mobile VLBI station fleet of trucks.

the ALMA construction, a submillimeter telescope is not so technically difficult to construct. However it is also true that a simple application of ALMA experience is not enough to realize the mobile radio telescope for such high frequency observations. In case of ALMA $12 \mathrm{~m}$ telescope, to obtain a large aperture for high frequency receiving, panels on rigid structure with actuator are adopted and successful performance is attained. But the weight of the ALMA telescope means it is too heavy to easily relocate and also complicated operations are required. The ALMA telescope is designed to fulfill universal functions, and as a result it is expensive, while our concept of the Caravan-submm mobile station is quite different from that of ALMA. The Caravan-submm mobile station must move around the Andes region. Its telescope must be transportable, and it must be easy to assemble and disassemble. To fulfill the requirements we adopt a multimirror system for our mobile station. Using a couple of lightweight dishes $2 \mathrm{~m}$ in diameter, we get a total aperture area corresponding to that of $4 \mathrm{~m}$ single dish. Use of lightweight small dishes brings us two advantages. The first is that individual small dishes are so light that we can easily transport them using the usual vehicles and can assemble them by hand and disassemble them after observations. Second, we can attain cost reduction when we use several small dishes instead of making a single dish with the same collecting area. In general, the cost of making a single dish increases more rapidly than is proportional to the square of its diameter. This is why we plan to use a multimirror system for the Caravan-submm mobile telescope (Figure 5).

2.3. Antenna by Metal Spinning Method. In order to achieve the cost reduction in making high precision antennas for receiving $230 \mathrm{GHz}$, we have examined the accuracy of the parabola surfaces made using the Japanese metal spinning method (Figure 6) and found that an accuracy as well as $60 \mu \mathrm{m}$ r.m.s. is easily obtained. Recently we confirmed that surface accuracies about $15 \mu \mathrm{m}$ r.m.s. were achieved by adding an annealing process in spinning processing (Figure 7).

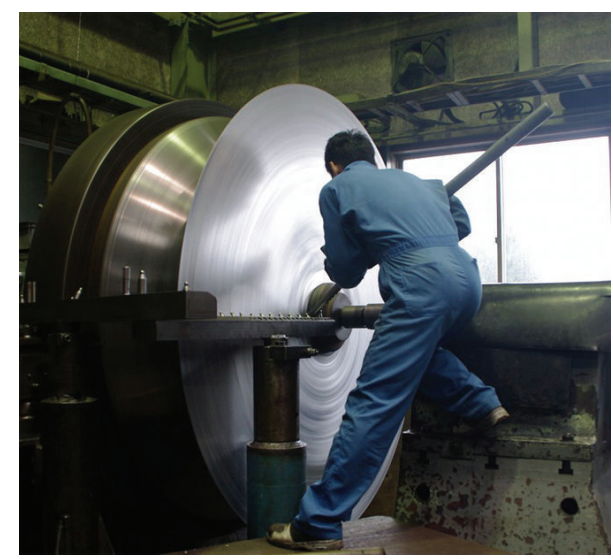

FIgURE 6: Japanese metal spinning method (Kitajima Shibori Seisakusho Co., Ltd.).

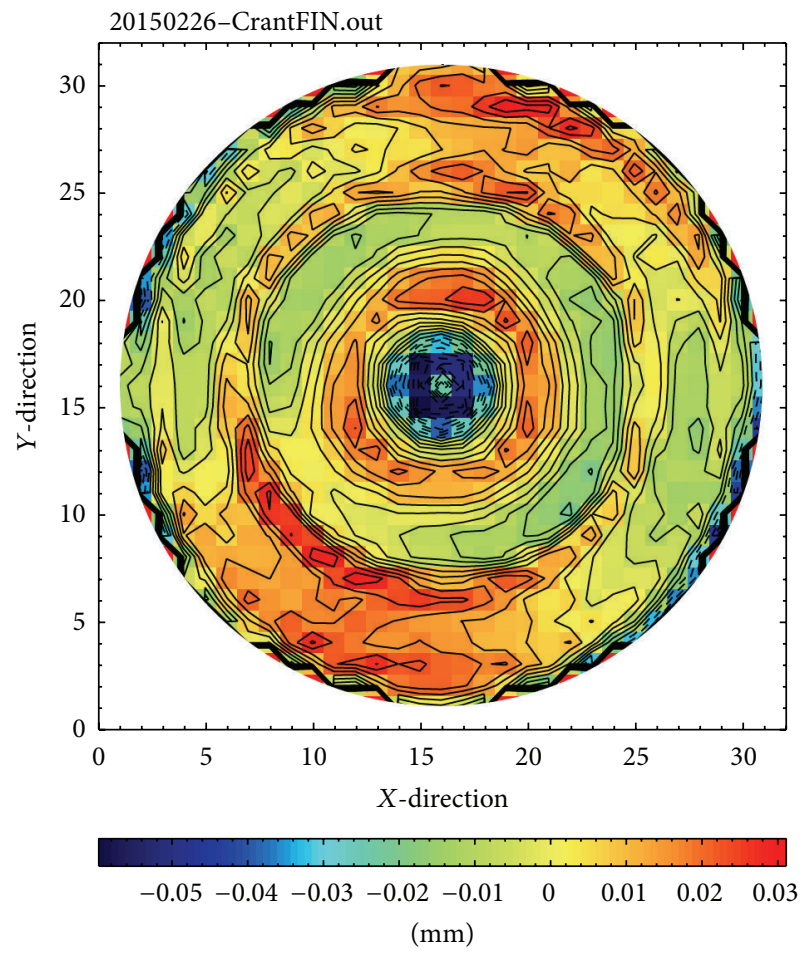

FIGURE 7: Surface accuracy about $15 \mu \mathrm{m}$ r.m.s. was achieved using a new $30 \mathrm{~cm}$ mold (surface accuracy: $4 \mu \mathrm{m}$ r.m.s.) by metal spinning method with adding an annealing process.

Now we have hopeful outlooks that cost reduced millimeter/submillimeter antennas as large as $2 \mathrm{~m}$ in diameter can be produced using the Japanese metal spinning method.

2.4. Site Survey. To observe with such high frequencies on the ground, high mountains such as the Andes are the best sites where the transparency of the millimeter/submillimeter wavelengths in the atmosphere is good. We performed a site survey in the Andes with the support of the ICRR. In order to 


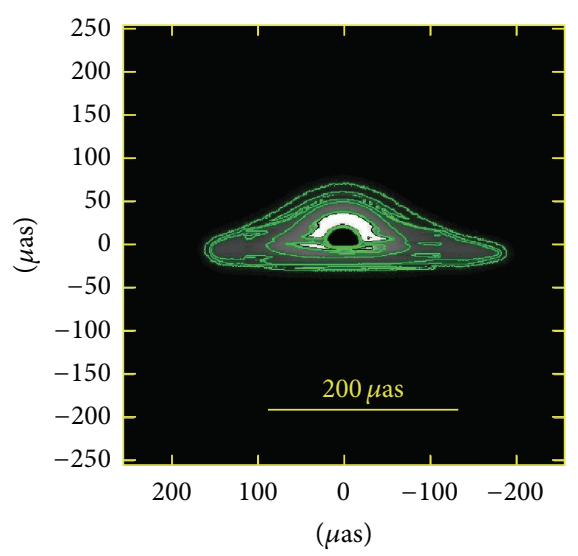

(a) Model image

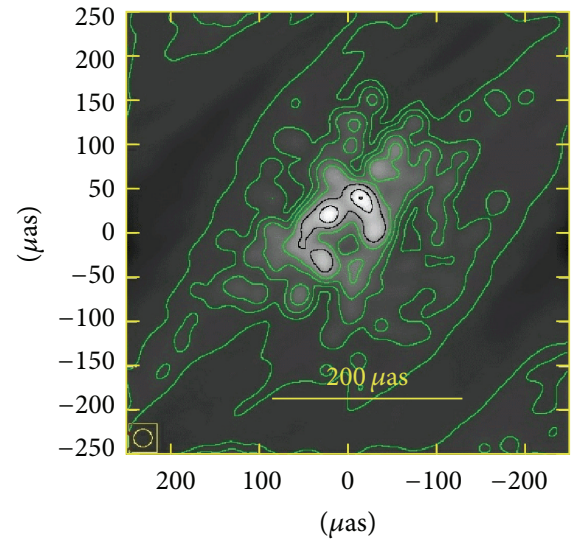

(b) Longer baselines only (without ALMA)

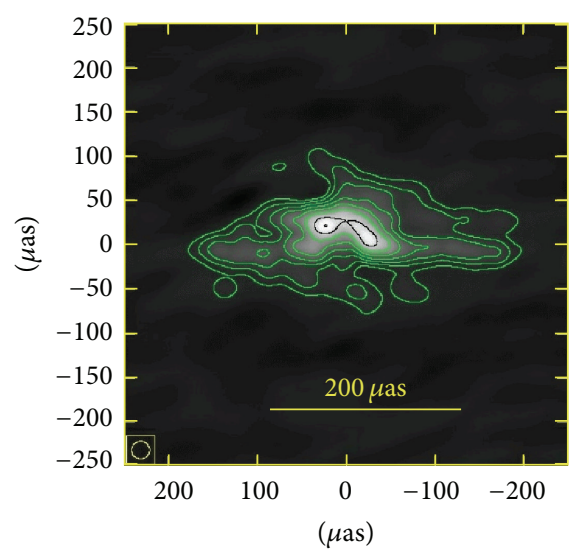

(d) Addition of Caravan (without ALMA)

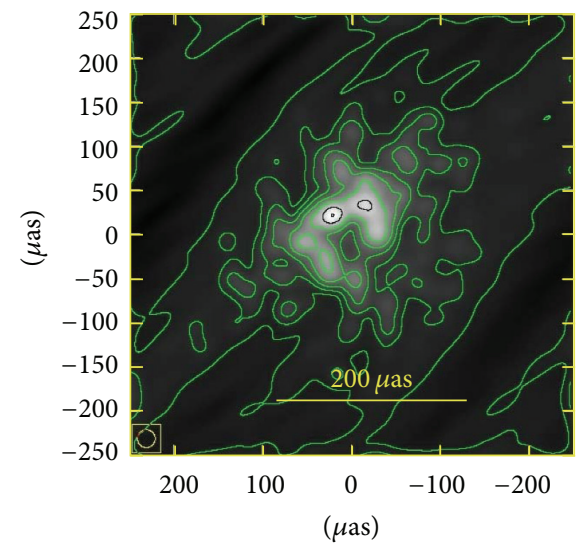

(c) Longer baselines only (with ALMA)

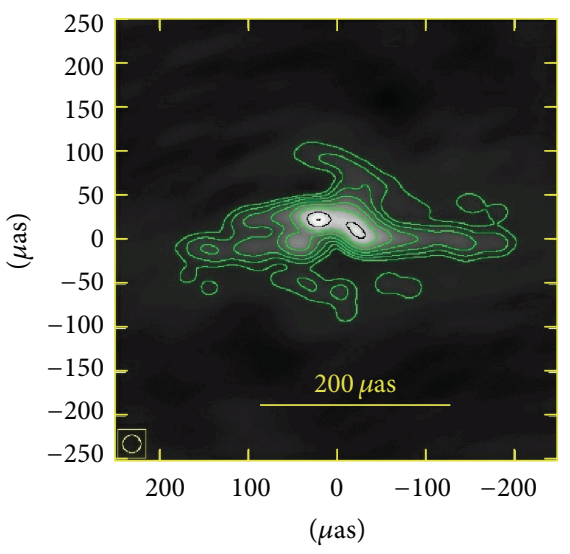

(e) Addition of Caravan (with ALMA)

FIGURE 8: Imaging simulation for $u-v$ coverage. (a) Model image of edge-on view RIAF accretion disk with $50 \mu$ as black hole shadow at the center. Total flux density is 3 Jy. (b) Result from only global longer baselines without phased-ALMA. (c) Result from only global longer baselines with phased-ALMA. (d) Result from combination of global longer baselines plus Caravan-submm array without phased-ALMA. (e) Result from combination of global longer baselines plus Caravan-submm array with phased-ALMA. Here we assumed $1024 \mathrm{MHz}$ recording bandwidth.

confirm the transparency at millimeter/submillimeter radio waves, measurement of the water vapor contents in the atmosphere should be done. We measured the water vapor contents at Huancayo Observatory (IGP) in Peru, Mount
Chacaltaya Laboratory (Universidad Mayor de San Andres) in Bolivia, and other places. We found the precipitable water vapor amounts in the rainy reason (February to March in 2015) were at least twice as high compared with those in 
the dry season (June to July in 2012). This means that the best season for millimeter/submillimeter VLBI observations is limited even in the high Andes.

2.5. System Operation. The Japanese Caravan-submm team will not only perform our observations in the Andes alone, but also take part in the worldwide observations. As a first step after constructing the Caravan-submm mobile station, we begin observations with other telescopes in the Andes. By changing observational positions of the Caravan-submm mobile station, we sample data from various baselines. Checking the fringe amplitude-baseline length relations, we investigate the first null point, from which position we can estimate the horizon size and the mass of black hole assuming simple image models (concerning the details of the method we explain in the next section). It is highly possible that at this stage we will obtain crucial evidence of a black hole horizon. We will take part in the global millimeter/submillimeter VLBI observation as soon as technically possible. From global millimeter/submillimeter VLBI observations of SgrA*, we can expect to obtain reliable imaging results of the black hole horizon.

2.6. Performance of Caravan-Submm. We performed further imaging simulations with changing array configurations and model images. Here we assumed a long baseline array contains seven stations, SMA (Hawaii, US), LLAMA (Argentina), LMT (Mexico), SMT (Arizona, US), CARMA (California, US), Plateau de Bure interferometer (Spain), and IRAM (Pico Veleta, Spain), and with/without phased-ALMA, while the Caravan-submm includes the two fixed stations at Huancayo, Peru, and Mt. Chacaltaya, Bolivia, with 13 different observing positions for the mobile station. Figure 8 shows a simulation result for SgrA*. First of all, resultant images have little difference between with and without the phased-ALMA. This is because $\mathrm{SgrA}^{*}$ is so bright at $230 \mathrm{GHz}$ that we can detect fringes of SgrA* with high signal to noise ratio even without ALMA. The essential role of phased-ALMA as a VLBI station lies in increasing the number of detectable sources with millimeter to submillimeter VLBI. Second, there is strong possibility that we fail to obtain a correct image with only global longer baselines like the present EHT array. Needless to say, it is also difficult to obtain a correct image with only shorter baselines like Caravan-submm array. It is from the combination of global longer baselines and Andes shorter baselines that we can obtain reliable images of SgrA* This conclusion is entirely consistent with the common sense in radio interferometry that image quality depends on how the $u-v$ plain can be covered.

\section{Data Analysis and Calibration}

In this section we introduce methods of data analysis and data calibrations. First, we mention a model fitting method using the relation between amplitude and projected baseline length that was frequently used in the old days and then two new methods we developed. The Slit Modulation Imaging (SMI) method is developed for detecting shorter time variations

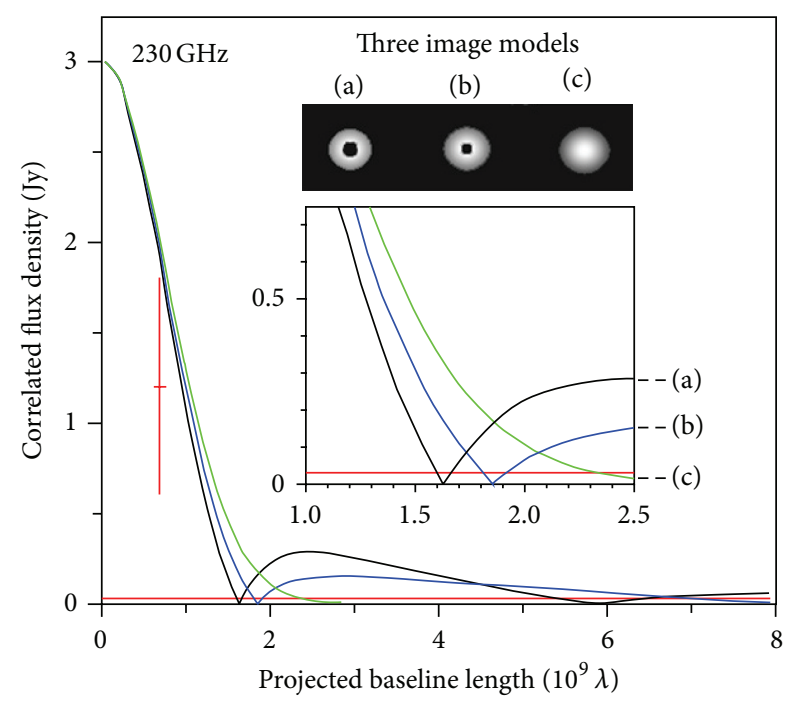

FIgUre 9: From Miyoshi et al. [8]: the visibility amplitudes of three image models as function of projected baseline: (a) the case of $M_{\mathrm{BH}}=3.7 \times 10^{6} \mathrm{M}_{\odot}$, (b) the case of $M_{\mathrm{BH}}=2.6 \times 10^{6} \mathrm{M}_{\odot}$, and (c) the case with no black hole or the scattering effect is still dominant. The functions of (a) and (b) have null value points that indicate the existence of the central black shadow. The $3 \sigma$ noise level of present engineering performance is shown by the red horizontal line. The red point with error bar is the visibility amplitude measured by Krichbaum et al. [9].

such as quasi periodic oscillation (QPO). The verification method of calibrations (VERICA) is the one to investigate the existence of residuals of systematic errors in calibrated data.

3.1. A Simple Analysis Using Amplitude u-v Distance Relation. When an array has limited coverage in the $u-v$ plane, instead of synthesis imaging, visibility analysis has frequently been performed in order to estimate the shape and size of the observed sources. In the early days of radio interferometers such methods were mainly used. Figure 9 shows visibility amplitude curves of three simple image models, (a) a simple Gaussian brightness without shadow, (b) a Gaussian with a shadow of $30 \mu \mathrm{as}\left(M_{\mathrm{BH}}=2.6 \times 10^{6} \mathrm{M}_{\odot}\right)$, and (c) a Gaussian with a shadow of $45 \mu$ as $\left(M_{\mathrm{BH}}=3.7 \times 10^{6} \mathrm{M}_{\odot}\right)$. For simplicity here we used point-symmetric images. A Gaussian brightness distribution also shows a Gaussian curve in the visibility amplitudes. If the shadow exists, the visibility function has null value points at some projected baseline length. The null value positions change with the size of the shadow. From the visibility amplitude function, we can distinguish whether the shadow exists or not. Further, because the null value points move according to the shadow size, we can estimate the shadow size, which also means we can measure the mass of the black hole from the null value positions. For measuring the correlated flux densities with $u-v$ distance, a small array composed of a few stations is sufficient. It is certain that the null value points will appear in visibility amplitude and projected baseline diagrams with other types of images. One of the typical ones is double sources like core and jet structures, which are frequently observed from other AGNs. 


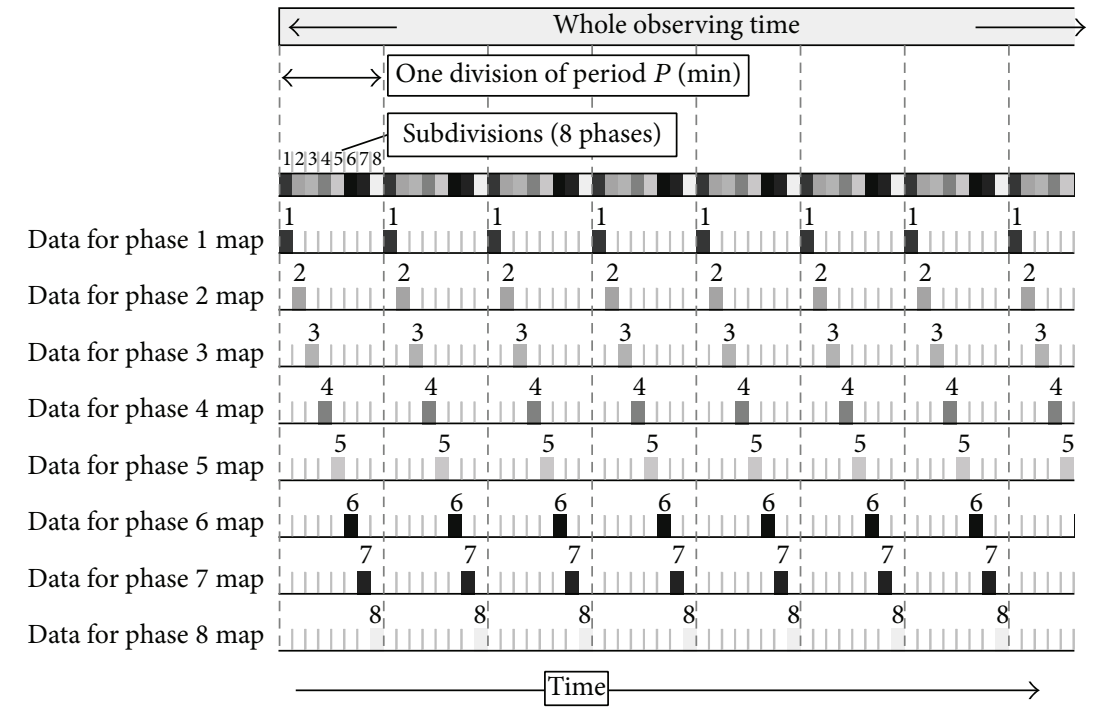

(a)
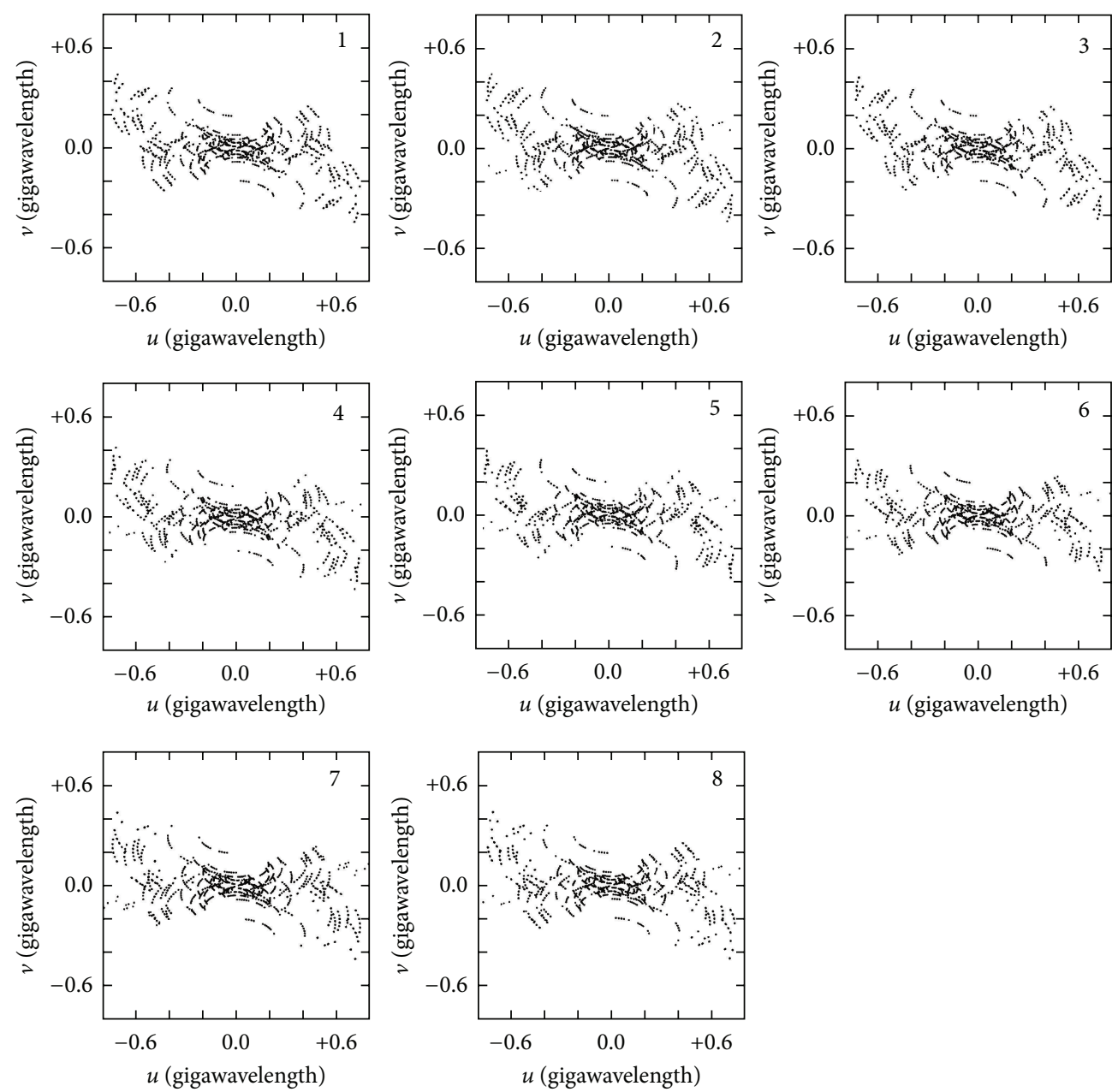

(b)
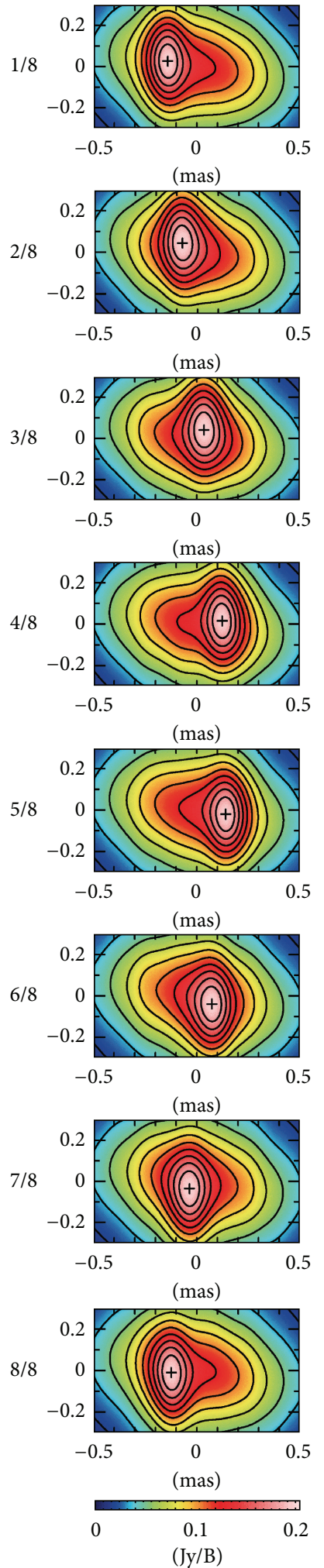

(c)

FIGURE 10: The SMI method. (a) Schematic diagram of data-sampling pattern in the SMI method. (b) $u$ - $v$ coverage of the 8-phase SMI maps for $\operatorname{SgrA}^{*}\left(\delta=-30^{\circ}\right)$ at $43 \mathrm{GHz}$ by VLBA. $N_{\text {div }}=8$ and $P_{\text {trial }}=16.8 \mathrm{~min}$. (c) A simulative result of the SMI method for model of moving point on fixed Gaussian at $\delta=-30^{\circ}$. The map area is 1 mas $(e-w) \times 0.5$ mas $(n-s)$. Contour levels are every $10 \%$ of the peak. Cross mark indicates the intensity peak position corresponding to the moving point position [10]. 


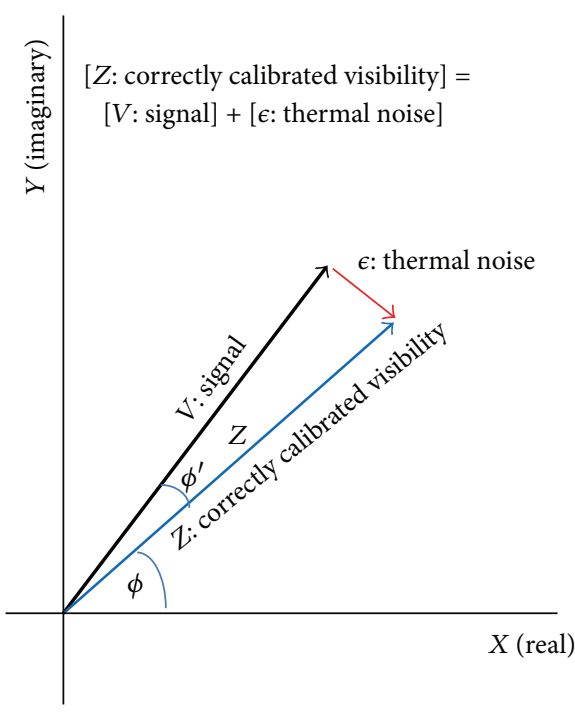

(a)

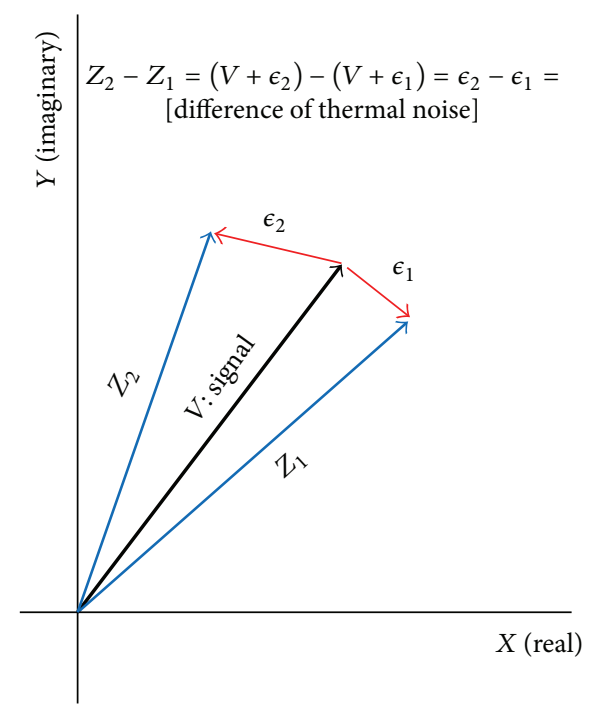

(b)

FIGURE 11: Relations of signal $V$, thermal noise $\epsilon$, and correctly calibrated visibility $Z$. If the calibration is perfectly performed, the differential visibility shows thermal noise feature. While the calibration is insufficient, namely, systematic errors still remain, the differential visibility shows different distributions from thermal ones.

In the case of SgrA*, there is no convincing result showing separated structures from previous VLBI observations. The VLBI image of SgrA* always shows a single structure. When an eruption happens at $\operatorname{SgrA}^{*}$, the double structure will appear. But the structure is a tentative one and will soon disappear. It will be easy to distinguish the reason for the existence of null value points in visibility amplitude whether from core and jet structures or from the darkness of a black hole shadow. Even if the SgrA* structure is not simple like the three models used here, we can limit the structure model from estimated mass and spin of the black hole in SgrA ${ }^{*}$ and the accretion disk model and shape of black hole shadow from ray-tracing calculations. Reliable image-model fitting to observed visibilities will certainly be possible to detect the black hole shadow in SgrA*. In the case of SgrA ${ }^{*}$ the first null point will appear at projected baseline length ranging from 1000 to $2000 \mathrm{~km}$. This is the reason why such shorter baselines of Caravan-submm are the key for detecting black hole horizon of SgrA* [14].

3.2. Slit Modulation Imaging (SMI) Method. The SMI method is for detecting shorter periodic structural changes of observed source from interferometric data. It is highly effective in detecting periodic change patterns whose period is shorter than the observing time span, from interferometric data [10]. The essence of the method lies in the sampling pattern of visibility data in time series. Figure 10(a) shows a schematic diagram of data selection from an observation time span. First we divide the whole observation time into several divisions with a trial period, $P_{\text {trial }}$, and each division is divided into subdivisions (n phase segments). Figure 10(a) shows the case of $N_{\text {div }}=8$. The $n$th phase SMI map is produced from the visibilities of the $n$th phase segments of all the divisions. Figure 10(b) shows the resultant $u-v$ coverage of each phase
SMI map. Thus, the SMI maps have very similar $u-v$ coverage, almost free from the influence of $u-v$ coverage differences. Unlike the analysis of time series of snapshot maps, there is no need to discuss the effect of different $u-v$ coverage. Figure 10(c) shows a simulation example that SMI method detects a periodic structure change: a $0.1 \mathrm{Jy}$ point source oscillates with period $P_{\text {change }}=16.8 \mathrm{~min}$ on a fixed elliptical Gaussian brightness distribution shape of SgrA* at $43 \mathrm{GHz}$. By changing $P_{\text {trial }}$ we can learn the periodic structure change of the source if it exists. As demonstrated, we can detect periodic structure change patterns with shorter periods than the observing time span.

3.3. VERICA (VERIfication Method of CAlibrations) Method. Verification of applied calibration onto visibility is important but has not been established well so far. We found such a method named VERICA recently. Because the source structure is unknown to us in general, we cannot predict how the signal $V$ behaves. However, if we can subtract the $V$ from the obtained visibility $Z$ and if systematic errors are removed by calibrations, the residual shows a pure thermal noise feature, which can be estimated from measured system temperatures and antenna performance. Differential visibilities between different video channels recorded at the same time with the same baseline (and each frequency is set to be nearly equal) correspond to such quantities. If the individual calibrations applied to the visibilities are precisely appropriate, the difference should statistically show a differential thermal noise distribution (Figure 11). Therefore, by checking distributions of differential visibilities, we can verify whether the applied calibrations onto visibility are proper or not without knowledge of observed source structure. From probability density functions of signal and thermal noise [27], we calculated its differential probability density 

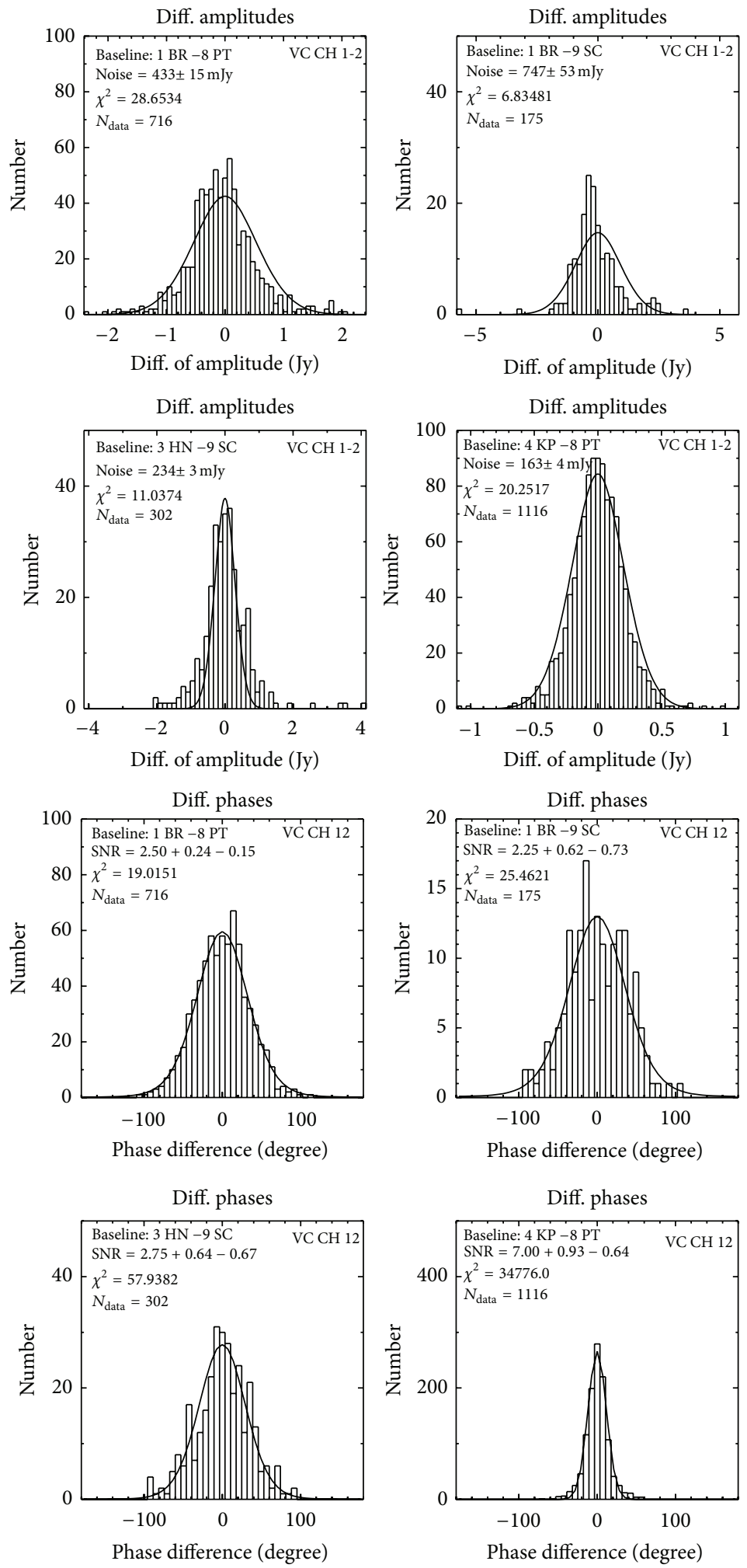

FIGURE 12: Examples of differential visibility distributions: from several baselines, BR-PT, BR-SC, HN-SC, and KP-PT. Every visibility is 10 sec integrations from VLBA $43 \mathrm{GHz}$ observations of SgrA* (code: BS131B). 


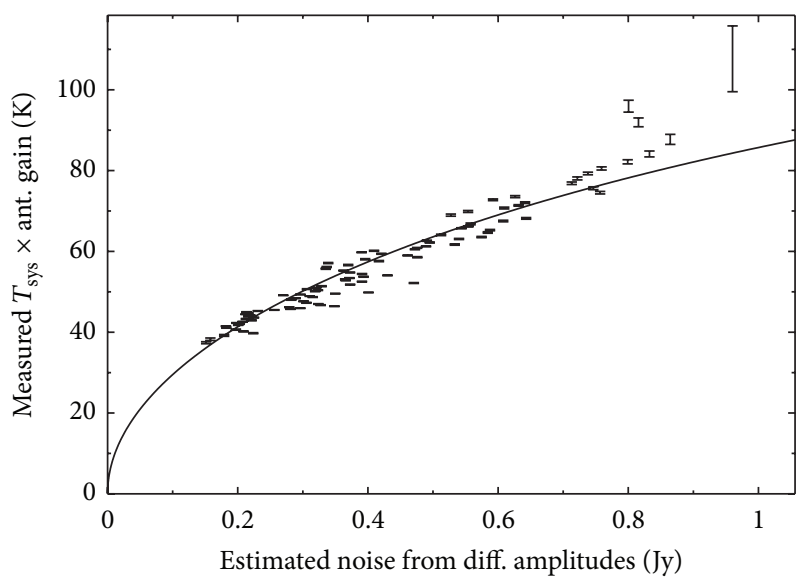

Figure 13: Estimated noise from fitting to differential visibility amplitude distribution and measured $T_{\text {sys }} \times$ gain. In this case, the calibrated data shows expected thermal noise features.

functions and developed this new method, VERICA [28]. Figure 12 shows examples of differential phase and amplitude distributions, respectively, from real VLBA $43 \mathrm{GHz}$ data of SgrA $^{*}$ [29]. Real distributions are fitted well to theoretical ones. From differential amplitude fitting, we can estimate the noise levels. We found the estimated noises are consistent with measured system noise and antenna gains (Figure 13). This means systematic errors are well removed by applied calibrations. We can judge whether the applied calibrations are proper or not by VERICA even when the observed source shows time variation during observations. This is why the differential visibility contains no component of source structure. At millimeter to submillimeter wave VLBI observations of SgrA*, we will encounter such situations in future, but using the VERICA method we can find good calibration solutions to be applied and obtain correct synthesis images. (Note: here each video channel is calibrated independently by Fringe-search and self-calibration. Thus the degrees of residual systematic errors are different in each data item after individual calibration. The differential visibility distributions between individually calibrated data show deviations from thermal noise ones if residual systematic errors still exist.)

\section{Competing Interests}

The authors declare that they have no competing interests.

\section{Acknowledgments}

The authors would like to thank Professor M. Tsuboi at Institute of Space and Astronautical Science, Japan Aerospace Exploration Agency, for technical discussion about multimirror systems. This work was supported in part by Grand-inAid for Scientific Research of JSPS (25610044 and 16K05302, Rohta Takahashi, and 23654071, Makoto Miyoshi and Takashi Kasuga).

\section{References}

[1] B. P. Abbott, R. Abbott, T. D. Abbott et al., "Observation of gravitational waves from a binary black hole merger," Physical Review Letters, vol. 116, Article ID 061102, 2016.

[2] C. T. Cunningham and J. M. Bardeen, "The optical appearance of a star orbiting an extreme KERR black hole," Astrophysical Journal, vol. 173, p. L137, 1972.

[3] J. M. Bardeen and C. T. Cunningham, "The optical appearance of a star orbiting an extreme Kerr black hole," Astrophysical Journal, vol. 183, pp. 237-264, 1973.

[4] C. T. Cunningham, "The effects of redshifts and focusing on the spectrum of an accretion disk around a Kerr black hole," Astrophysical Journal, vol. 202, pp. 788-802, 1975.

[5] J.-P. Luminet, "Image of a spherical black hole with thin accretion disk," Astronomy and Astrophysics, vol. 75, no. 1-2, pp. 228-235, 1979.

[6] J. Fukue and T. Yokoyama, "Color photographs of an accretion disk around a black hole," Publications of the Astronomical Society of Japan, vol. 40, no. 1, pp. 15-24, 1988.

[7] R. Takahashi, "Black hole shadows of charged spinning black holes," Publications of the Astronomical Society of Japan, vol. 57, no. 2, pp. 273-277, 2005.

[8] M. Miyoshi, S. Kameno, J. K. Ishitsuka, Z.-Q. Shen, R. Takahashi, and S. Horiuchi, "An approach detecting the event horizon of Sgr A*," Publication of the Astronomical Society of Japan, vol. 10, pp. 15-23, 2007, http://www.nao.ac.jp/aboutnaoj/reports/publications-naoj.html, http://www.nao.ac.jp/contents/about-naoj/reports/publications-naoj/10-1234-2.pdf.

[9] T. P. Krichbaum, D. A. Graham, A. Witzel et al., "VLBI observations of the galactic center source SGR A* at $86 \mathrm{GHz}$ and $215 \mathrm{GHz}$," Astronomy and Astrophysics, vol. 335, pp. L106L110, 1998.

[10] M. Miyoshi, "Slit-modulation imaging method for detecting any periodic structural change in interferometric data," Publications of the Astronomical Society of Japan, vol. 60, no. 6, pp. 1371-1386, 2008.

[11] A. M. Ghez, S. Salim, N. N. Weinberg et al., "Measuring distance and properties of the milky way's central supermassive black hole with stellar orbits," The Astrophysical Journal, vol. 689, no. 2, pp. 1044-1062, 2008.

[12] Z.-Q. Shen, K. Y. Lo, M.-C. Liang, P. T. P. Ho, and J.-H. Zhao, "A size of $\sim 1$ AU for the radio source Sgr A* at the centre of the Milky Way," Nature, vol. 438, no. 7064, pp. 62-64, 2005.

[13] H. Falcke, F. Melia, and E. Agol, "Viewing the shadow of the black hole at the Galactic center," The Astrophysical Journal, vol. 528, no. 1, pp. L13-L16, 2000.

[14] M. Miyoshi, J. K. Ishitsuka, S. Kameno, Z.-Q. Shen, and S. Horiuchi, "Direct imaging of the massive black hole, SgrA*," Progress of Theoretical Physics Supplement, vol. 155, pp. 186-189, 2004.

[15] S. S. Doeleman, J. Weintroub, A. E. E. Rogers et al., "Eventhorizon-scale structure in the supermassive black hole candidate at the Galactic Centre," Nature, vol. 455, pp. 78-80, 2008.

[16] K. Gebhardt, J. Adams, D. Richstone et al., "The black hole mass in M87 from GEMINI/NIFS adaptive optics observations," The Astrophysical Journal, vol. 729, no. 2, article 119, 13 pages, 2011.

[17] G. C. Bower, H. Falcke, R. M. Herrnstein, J.-H. Zhao, W. M. Goss, and D. C. Backer, "Detection of the intrinsic size of sagittarius A* through closure amplitude imaging," Science, vol. 304, no. 5671, pp. 704-708, 2004. 
[18] S. S. Doeleman, Z.-Q. Shen, A. E. E. Rogers et al., "Structure of Sagittarius $A^{*}$ at $86 \mathrm{GHz}$ using VLBI closure quantities," Astronomical Journal, vol. 121, no. 5, pp. 2610-2617, 2001.

[19] K. Y. Lo, Z.-Q. Shen, J.-H. Zhao, and P. T. P. Ho, "Intrinsic size of Sagittarius A* 72 Schwarzschild radii," The Astrophysical Journal, vol. 508, no. 1, pp. L61-L64, 1998.

[20] G. C. Bower, D. C. Backer, and R. A. Sramek, "VLBA observations of astrometric reference sources in the galactic center," The Astrophysical Journal, vol. 558, no. 1, pp. 127-132, 2001.

[21] M. D. Johnson, V. L. Fish, S. S. Doeleman et al., "Resolved magnetic-field structure and variability near the event horizon of Sagittarius A*," Science, vol. 350, no. 6265, pp. 1242-1245, 2015.

[22] G. C. Bower, H. Falcke, D. C. Backer, and M. Wright, The Central Parsecs of the Galaxy, vol. 186 of ASP Conference Series, Astronomical Society of the Pacific, 1999.

[23] R. Ichikawa, A. Ishii, H. Takiguchi et al., "Present status and outlook of compact VLBI system development for providing over $10 \mathrm{~km}$ baseline calibration," in Proceedings of the 8th NICT IVS TDC Symposium, Kashima, Japan, February 2009, http:// www2.nict.go.jp/aeri/sts/stmg/ivstdc/news_30/pdf/tdcnews_30 .pdf.

[24] S. Matsuzaka, M. Tobita, Y. Nakahori, J. Amagai, and Y. Sugimoto, "Detection of Philippine Sea plate motion by very long baseline interferometry," Geophysical Research Letters, vol. 18, no. 8, pp. 1417-1419, 1991.

[25] Y. Fukuzaki, K. Wada, R. Kawabata, M. Ishimoto, and T. Wakasugi, "First geodetic result of ishioka VGOS antenna," in Proceedings of the 14th NICT TDC Symposium, 35, p. 1, TDC News, Kashima, Japan, June 2015, http://www2.nict.go.jp/aeri/ sts/stmg/ivstdc/news_35/tdc_news35.pdf.

[26] K. Takefuji and MICE2015 Team, "First Japanese $230 \mathrm{GHz}$ VLBI experiment by MICE2015 team," in Proceedings of the 14th NICT TDC Symposium, p. 22, Kashima, Japan, June 2015, http:// www2.nict.go.jp/aeri/sts/stmg/ivstdc/news_35/tdc_news35.pdf.

[27] A. R. Thompson, J. M. Moran, and G. W. Swenson, Interferometry and Synthesis in Radio Astronomy, John Wiley \& Sons, New York, NY, USA, 2nd edition, 2001.

[28] M. Miyoshi, "A new verification method of applied calibrations onto visibility data," in Proceedings of the 13th NICT TDC Symposium, p. 3, Kashima, Japan, June 2014, http://www2.nict .go.jp/aeri/sts/stmg/ivstdc/news_34/pdf/pctdc_news34.pdf.

[29] M. Miyoshi, Z.-Q. Shen, T. Oyama, R. Takahashi, and Y. Kato, "Oscillation phenomena in the disk around the massive black hole Sagittarius A," Publication of the Astronomical Society of Japan, vol. 63, no. 5, pp. 1093-1116, 2011. 

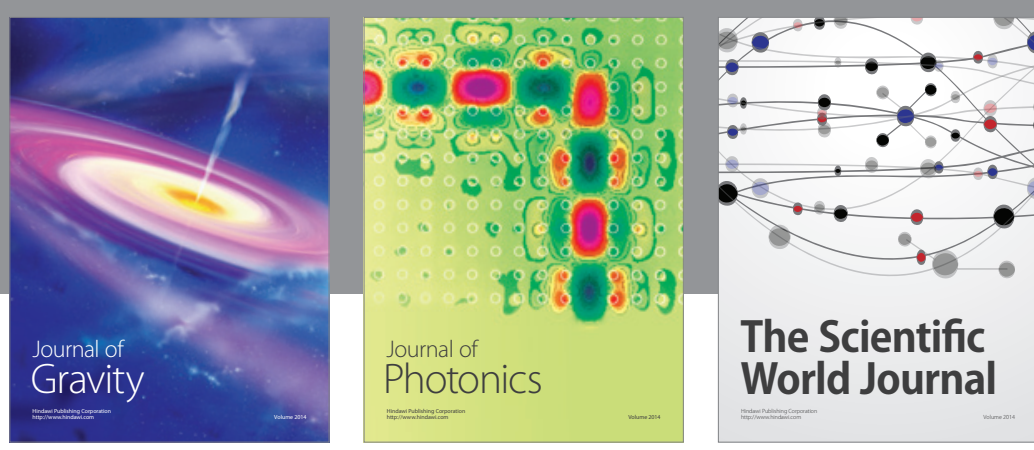

The Scientific World Journal
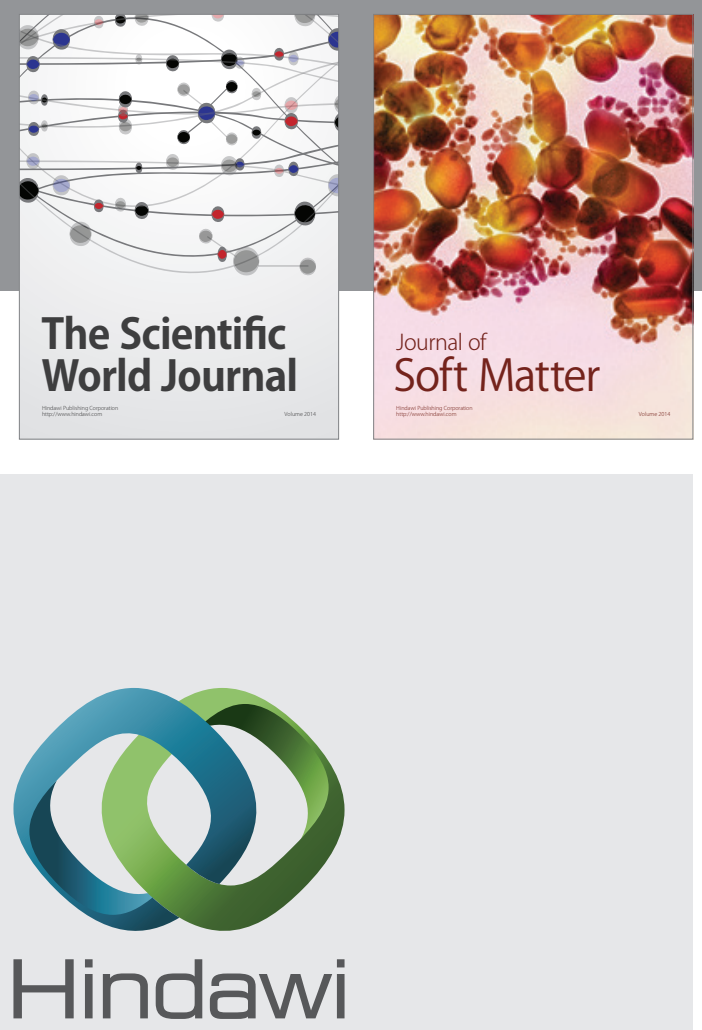

Submit your manuscripts at

http://www.hindawi.com

nternational Journal of

Statistical Mechanics
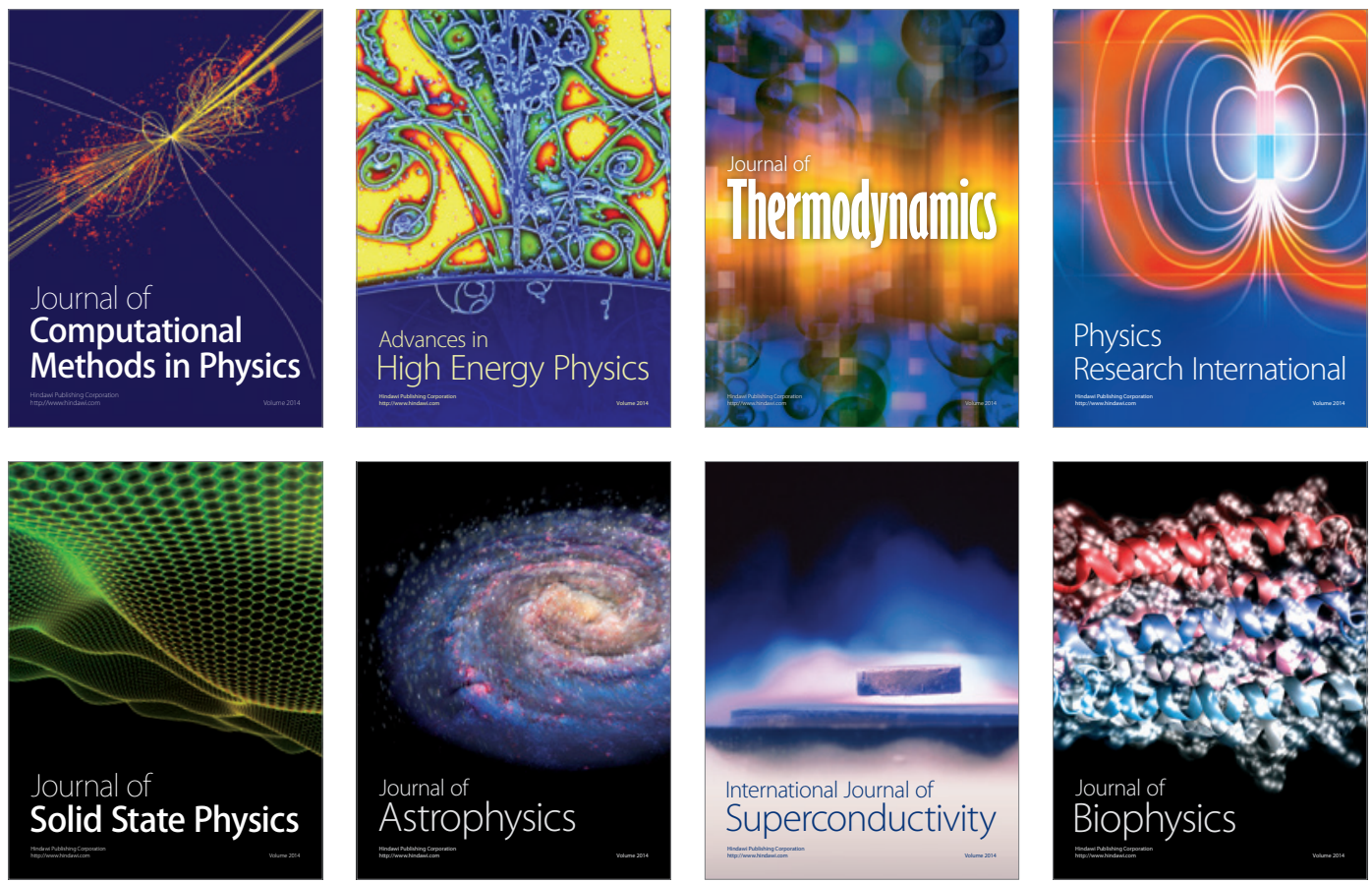
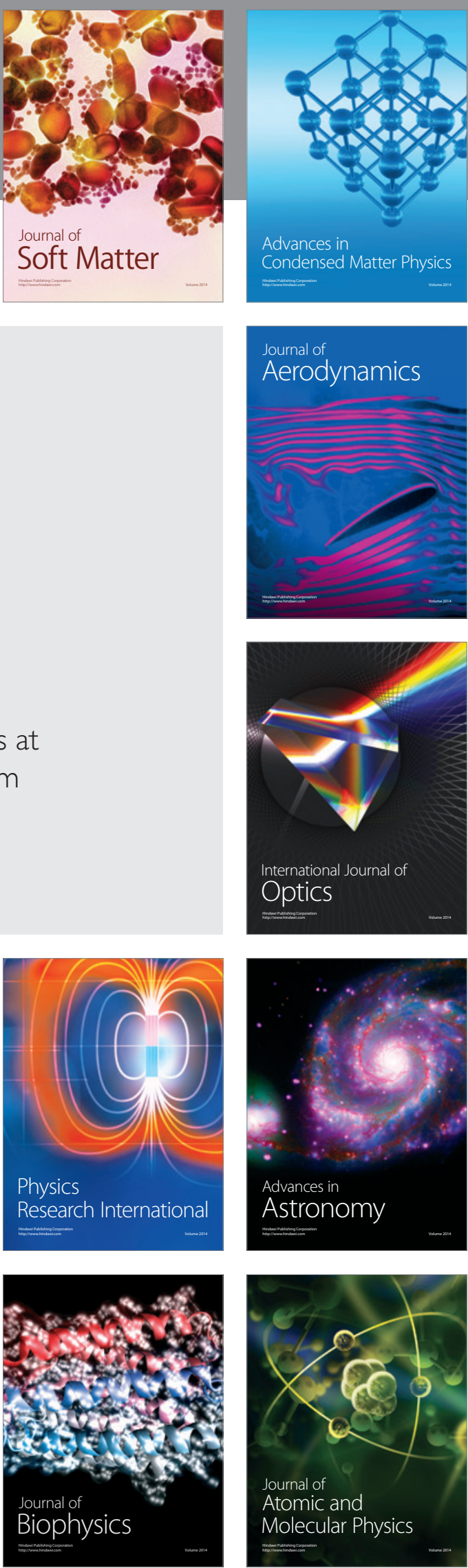